\title{
Chemical genetics: tailoring tools for cell biology
}

\author{
Thomas U. Mayer \\ Max Planck Institute of Biochemistry, Department of Cell Biology, Am Klopfersitz 18a, D-82152 Martinsried, Germany
}

\begin{abstract}
Chemical genetics is a research approach that uses small molecules as probes to study protein functions in cells or whole organisms. Here, I review the parallels between classical genetic and chemicalgenetic approaches and discuss the merits of small molecules to dissect dynamic cellular processes. I then consider the pros and cons of different screening approaches and specify strategies aimed at identifying and validating cellular target proteins. Finally, I highlight the impact of chemical genetics on our current understanding of cell biology and its potential for the future.
\end{abstract}

It is an omnipresent challenge for every cell biologist to dissect a complex biological process within a cellular system. In general, it is not only the number of the participating components but also the complexity of their regulation and interactions that constitute this daunting task. Historically, genetic approaches have proven to be extremely powerful in elucidating the principles of a wide range of biological processes such as cell division in the budding yeast Saccharomyces cerevisiae [1], programmed cell death and organ development in the nematode Caenorhabditis elegans [2] or early embryonal development in the fruitfly Drosophila melanogaster [3]. These famous screens, honored by the Nobel Prize, are classical examples of forward-genetic approaches, which can be outlined by a three-step procedure: first, the genome of the chosen organism has to be mutagenized in a random fashion (e.g. EMS, X-ray, transposons), then the desired phenotype has to be detected within the large collection of generated mutants and, finally, the mutated gene that underlies the phenotype has to be identified. Whereas the forward-genetic method works its way from the desired phenotype 'forward' to the underlying mutated gene, reverse-genetics elucidates the function of a protein of interest by analyzing the phenotype when the encoding gene is mutated. In these studies, the introduced mutations can either affect the activity of the chosen protein (e.g. site-specific mutations) or entirely suppress its expression (e.g. 'knockout' mutations).

What is chemical genetics and why make use of it? The term 'chemical genetics' has been coined for an approach that uses small organic molecules (see Fig. 1)

Corresponding author: Thomas U. Mayer (mayer@biochem.mpg.de). as probes to study protein functions in cultured cells or whole organisms. In accordance with the aforementioned genetic approaches, we can distinguish between forwardand reverse-chemical genetics. A forward-chemical genetic screen - like its genetic counterpart - can be subdivided into three discrete steps: first, the organism or cell type of interest has to be treated with a diverse collection of small molecules. This step mirrors the introduction of random genetic mutations in the forwardgenetic approach. The goal of the second step is to identify the desired phenotype within the large collection of compound-treated organisms or cells and, finally, the cellular molecules (e.g. proteins, lipids, sugars) targeted by the bioactive compounds have to be identified. Similar to the genetic approach, the starting point for reverse chemical genetics is a protein of interest for which bioactive ligands are searched out of a large pool of small molecules.

An interesting combination of reverse genetics with chemistry is provided by a recent approach pioneered by Shokat and colleagues [4]. The underlying idea of their method is to genetically engineer a mutant nucleotide binding protein (e.g. kinase, motor protein), which becomes sensitive to a nucleotide analog but retains almost normal enzymatic activity in the presence of the wild-type nucleotide. In this way, the mutant allele can be inhibited by the nucleotide analog in a cellular context, whereas all other wild-type nucleotide binding proteins remain essentially unaffected (see below).

The genetic approach is powerful in that it can readily identify gene products involved in a specific process and then mutate the encoding gene with complete specificity within a complex cellular context. However, there are situations in which small molecules might be more useful. First, in many cases, one and the same small molecule can be applied to study a process of interest in a wide range of organisms, whereas the genetic approach logically demands that the desired genetic modification has to be regenerated every time the experimental system is switched. For example, brefeldin A was instrumental to study transport processes from the endoplasmic reticulum (ER) to Golgi in organisms as different as yeasts, plants and mammalian cells [5].

Second, most mutations are not conditional, which means that the activity of the protein of interest cannot be turned on or off at will. And even if conditional alleles are available (e.g. temperature-sensitive mutations or mutant alleles under the control of inducible promoters), it typically 


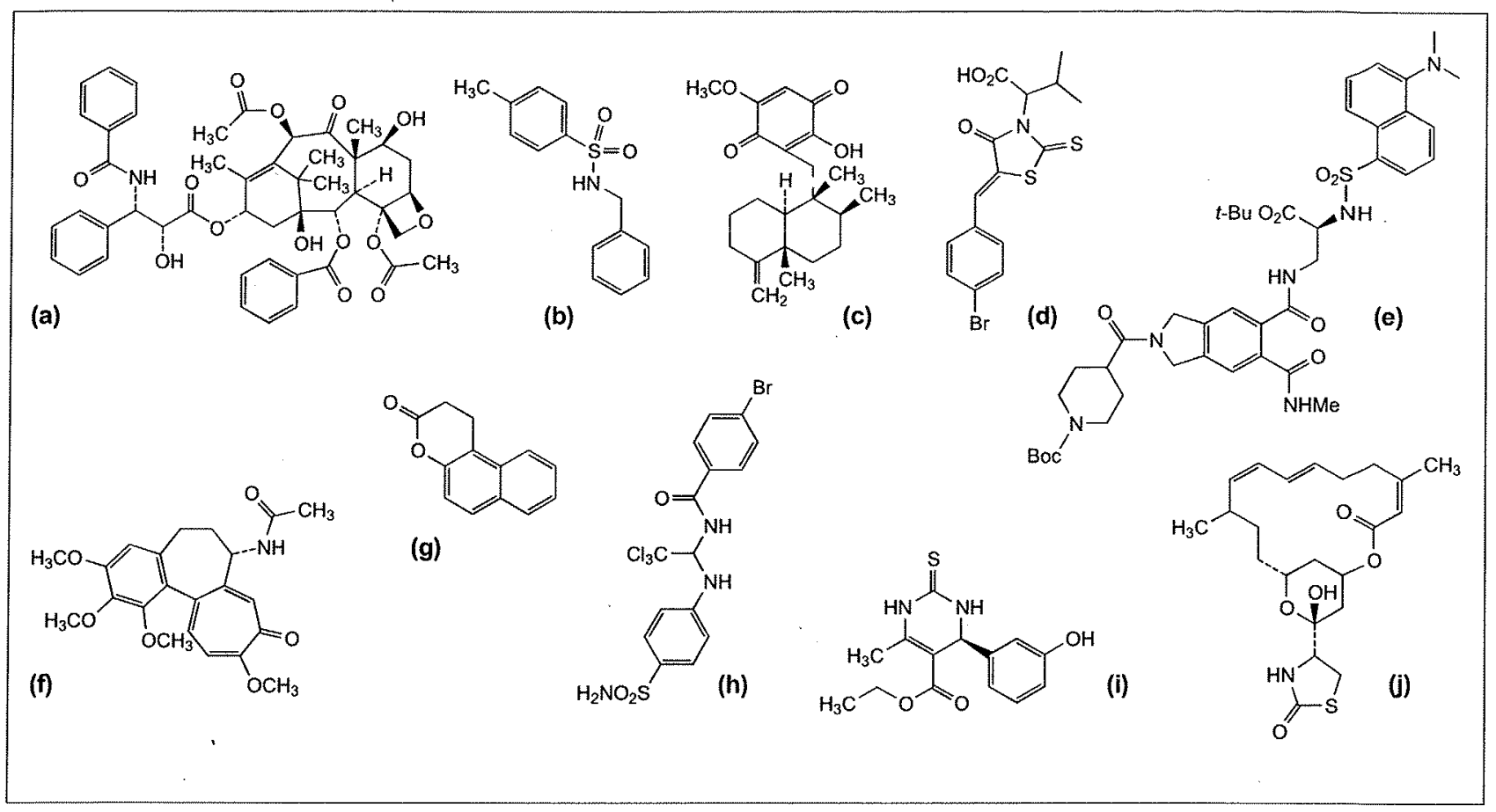

Fig. 1. Chemical structures of smalt molecules discussed in this review: (a) taxol, (b) BTS, (c) ilimaquinone, (d) BH3I-1, (e) IIA4B20, (f) colchicine, (g) splitomicin, (h) synstab A, (i) monastrol, (j) latrunculin A. In the context of this review, the term 'small molecules' is applied to all organic compounds with a molecular mass of less than 1500 Da, which have been either chemically synthesized or isolated from biological extracts. (For a review on peptide and peptidomimetic inhibitors, see [66]).

requires hours to change the protein complement of a cell. This lack of temporal control substantially limits the use of genetic approaches to study dynamic processes. By contrast, small molecules change directly the way proteins work, thereby representing tools with a uniquely high temporal resolution, capable of dissecting dynamic cellular processes. Notably, small molecules bear the potential to gain insights into fundamental biological processes not only by studying compound-treated cells but also by observing cells released from compound treatments. For example, the role of the microtubule-organizing center (MTOC) in nucleating microtubules was initially recognized by studies of cultured cells recovering from treatment with the microtubule poison colcemid [6].

of particular interest, chemical genetics is predestined to pave the way for interdisciplinary research uniting biology, chemistry, pharmacology and medicine. In this way, basic research has the potential to create promising leads for the development of new therapeutic drugs. Of particular note, small molecules targeting microtubules represent impressive examples of synergies between basic research and drug development: colchicine was first used over 2000 years ago in the form of preparations of the meadow saffron Colchicum autumnale to treat pain associated with gout attacks. After the isolation of (-)-colchicine as the active constituent of the plant by the French chemists Pelletier and Caventon in 1820, this compound led to the discovery of tubulin by Taylor and colleagues in the late 1960s [7]. Today, colchicine is still one of the more effective treatments for gout, and, at the same time, it is a versatile probe to study microtubule functions in diverse cell-biological processes such as cell migration, trafficking of intracellular vesicles or mitosis [8]. Taxol, the active compound from the bark of the Pacific yew tree (Taxus brevifolia), was identified in 1971 as a substance with antitumor activity and is today an approved drug to treat breast and ovarian cancer [9]. Unlike most other microtubule poisons (e.g. colchicine, nocodazole, the Vinca alkaloids), taxol actually promotes the polymerization of microtubules in vitro and in vivo $[10,11]$ and therefore plays an essential role in almost all experiments requiring artificial microtubule polymerization (e.g. purification of microtubule-associated proteins or studies on microtubulebased motor proteins in vitro $[12,13]$ ).

\section{The foundation of chemical screens:} small-molecule libraries

In the past, biologically active small molecules have been mainly identified in complex biological mixtures derived from plants, marine sponges, fungi or bacteria. Obviously, this approach bears the risk that the initially observed activity is caused by the synergistic effect of numerous compounds rather than by a single small molecule. One way to circumvent this pitfall is to use large collections of defined, structurally diverse small molecules. However, until recently, this approach was restricted to pharmaceutical companies, who have assembled chemical libraries in the order of hundreds of thousands of compounds over the past century.

The enormous progress achieved in the field of combinatorial chemistry (see Box 1) [14], which has allowed the rapid production of a large number of chemically diverse molecules, was an important prerequisite to make chemical libraries available to academic researchers. As a result, a 


\section{Box 1. Definition of terms}

Combinatorial chemistry: A strategy to synthesize multiple com pounds in one step by combining variations of the different building blocks in the same reaction mixture. In this way, a single reaction carried out with, for example, five derivatives of reactant $A$ and five derivatives of reactant $B$ can yield 25 structurally related products.

Whole-cell immunoassay. Chemiluminescent based method to detect an antigen of interest in whole cells fixed in microtitre plates using a specific primary antibody and a secondary antibody coupled to horseradish peroxidase.

Phage display: A method to individually display peptides and proteins on the surface of a small bacterial virus called a phage. Interactions between the displayed peptides or proteins and the small molecule of interest can be detected by using a traceable derivative of the compound (radioactive or otherwise tagged eg biotin)

Protein microarray: High-throughput device to detect proteinprotein, protein-substrate or proteln-small-molecule interactions. Protein microarrays are created by the covalent attachment of peptides or proteins on glass supports. The identification of small molecule interaction partners follows the same strategy depicted for phage display,

Three-hybrid system: this is based on a synthetic hybrid molecule composed of a compound with a known binding partner (e.g, dexamethasone, which binds to the hormone-binding domain of the glucocorticoid receptor) and the small molecule of interest (Fig. I).
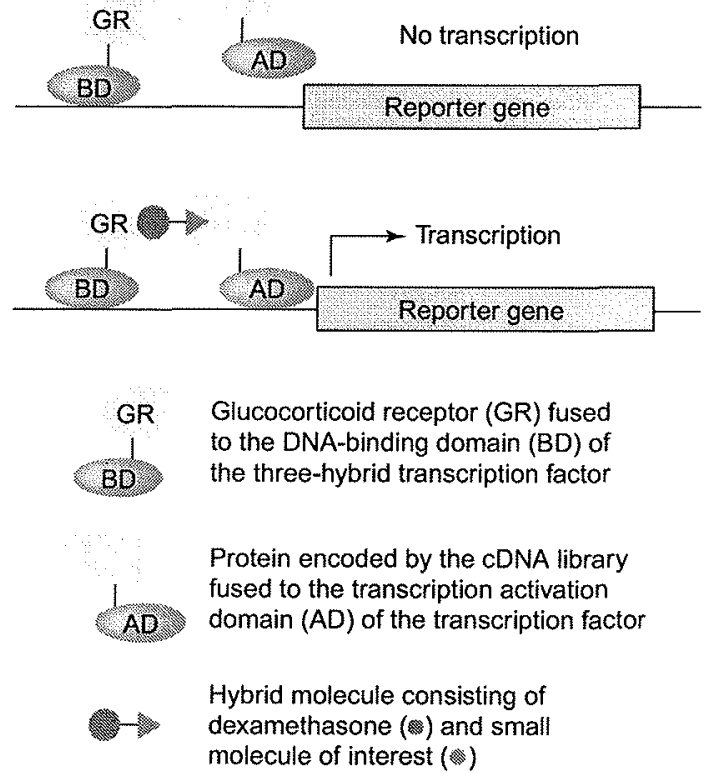

Fig. 1.

constantly growing number of suppliers offer libraries of small molecules [15] that are suitable for use with screening platforms typically found in academia. Today, one of the biggest challenges for anyone interested in chemical genetics is to pick a library that fits best the intended screens, the available screening platform and, of course, the personal budget available. Many suppliers offer "preselected' collections of 'drug-like molecules', known as 'pharmacophore' libraries [16]. Each of these commercially available libraries displays a high degree of structural diversity, whereas the individual compounds typically fulfil the following criteria: they are likely to cross cell membranes, they contain substructures resembling known bioactive molecules and they do not contain 'functional groups' (e.g. highly reactive groups) that are likely to cause cytotoxic effects. In addition to commercial suppliers, there are also nonprofit organizations offering small-molecule libraries such as the 'diversity set' of the National Cancer Institute, the 'NINDS collection' of the National Institute of Neurological Disorders and Stroke and the 'natural product pool' of the Hans-Knoell Institute [17].

\section{The road to success: designing a suitable high-throughput screen}

Given the challenge that typically tens of thousands of compounds have to be analyzed to find the desired bioactive molecules, it is self-evident that the intended screen has to be robust, automated as far as possible and miniaturized to an extent compatible with the 96-, 384or even 1536-well format. In the process of designing a suitable screening approach, the first and major decision to be made is between a 'pure protein' and a 'cell-based' assay (see Fig. 2). Pure-protein screens are performed in vitro through binding or enzymatic assays using mainly optical readouts such as luminescence, absorbance or fluorescence (e.g. fluorescence intensity, fluorescence polarization, fluorescence resonance energy transfer [18]). By contrast, cell-based assays ensure that the target protein exists in its physiological context and that the identified molecules are membrane-permeable. A common cell-based approach is the reporter-gene assay, where the accumulation of an easily detectable enzymatic activity (e.g. luciferase) depends on the activity of the target protein (e.g. a transcriptional regulator). Alternatively, cell-based approaches can make use of functional readouts (e.g. cell viability) or of "wholecell immunoassays' (see Box 1), a luminescence-based method that uses a suitable antibody to detect an epitope in cells whose occurrence or disappearance can be used as a readout for a specific process of interest [19]. These latter two cell-based methods are not biased towards one particular protein but are conceptually broader, offering the potential to find compounds for poorly characterized or even unknown targets.

Microscope-based approaches represent a new and exciting trend in cell-based screening towards acquiring more complex data. Unlike the aforementioned methods, this approach is capable of detecting subtle morphological changes (see Fig. 3). Moreover, microscope-based methods have the great advantage that they allow the collection not only of data on the specific process under investigation but also data on effects indirectly influencing the process of interest. For example, in a screen for inhibitors of cell migration (see Fig. 3), the total cell count can be applied to identify and sort out cytotoxic compounds, whose inhibitory activity on cell migration is indirectly caused by the fact that the cells are dying. Recently, this type of approach, which obviously demands the use of automated microscopes and image-analysis software [20], has been successfully applied to identify small molecules interfering 


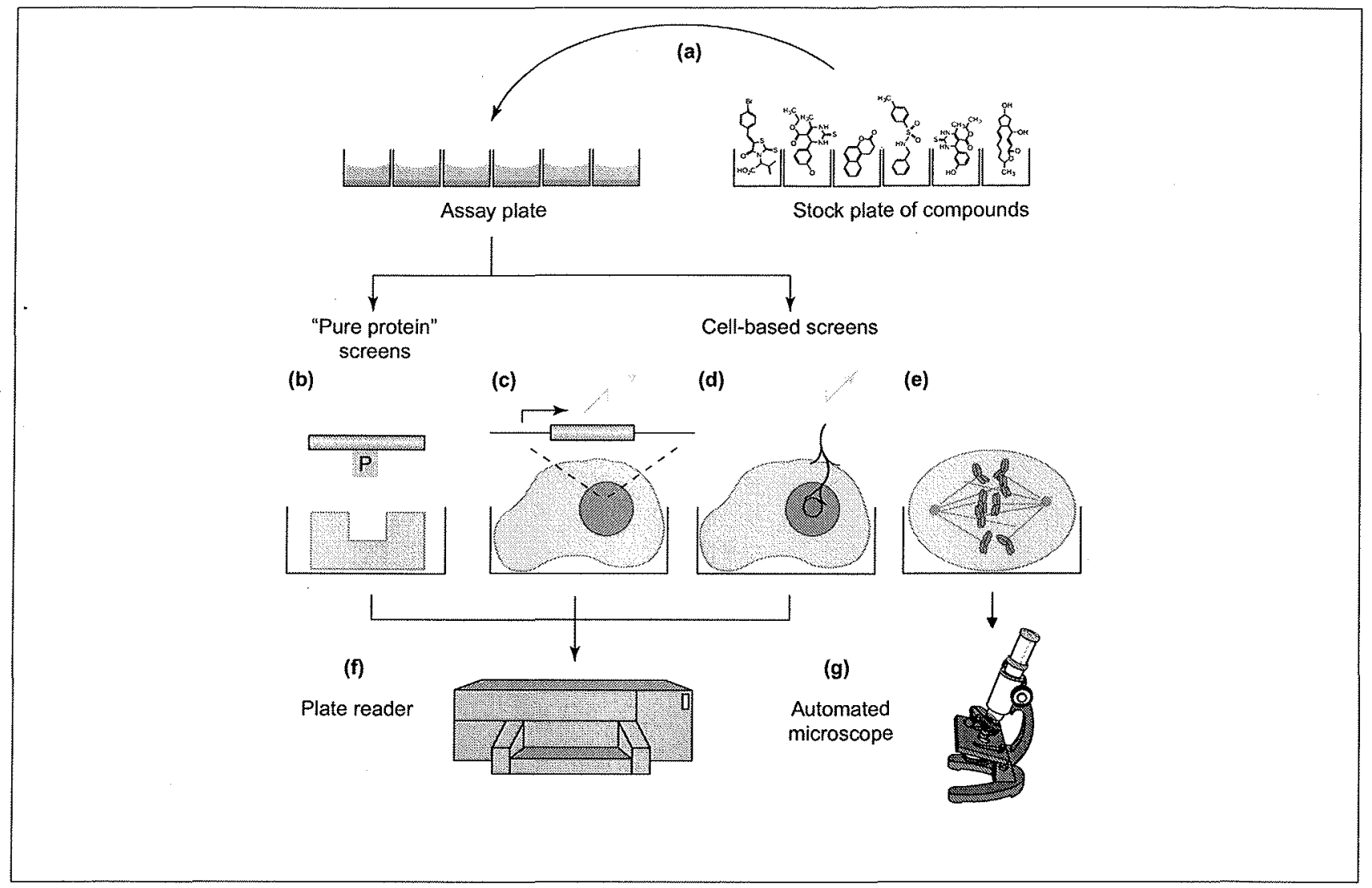

Fig. 2. Small-molecule screens can be performed in vitro and in vivo. (a) A small volume of a single compound is added to each well of an assay plate containing protein solution or the cell line of interest. (b) Pure-protein assays can be applied to identify compounds modulating the activity of the protein of interest in vitro (e.g. the release of phosphate into the solution can be used as readout for the activity of a phosphatase). (c) Compounds targeting transcriptional regulators (e.g. p53) can be identified by measuring the expression level of a reporter gene (e.g. luciferase). (d) Whole-cell immunoassays apply specific antibodies to detect posttranslational changes within cells. (e) Microscope-based assays detect subtle morphological changes within cells - e.g. spindle morphology. (f) Plate readers measure optical signals (e.g. luminescence, absorbance) obtained by quantitative assays $(b-d)$. $(g)$ Morphological changes are detected by automated microscopes designed to acquire images at different wavelengths and at various positions within each well of a microtitre plate.

with a wide range of biological processes, including embryonic development [21], cell differentiation [22] and the transport of intracellular vesicles [23]. Table 1 highlights examples of bioactive compounds that have been recently identified via pure-protein or cell-based screens.

\section{Target identification and validation}

Target identification represents a major challenge for all forward-chemical genetic screens (see above). In the past, biochemical purifications have been the method of choice for target identification. The two main routes are either to follow a traceable (radioactive or otherwise tagged - for example - biotin) derivative of the bioactive compound during fractionation of cell extracts or to immobilize the compound via a chemical linker on a solid phase support, followed by affinity purification of the cellular target [24]. Biochemical methods have been exceptionally successful in target identification (see Table 2). However, this approach reaches its limits when chemical modifications of the compound are not compatible with its biological activity or

Table 1. Pure-protein and cell-based small-molecule screens have yielded a wide range of bioactive compounds

\begin{tabular}{|c|c|c|c|c|}
\hline Compound name & Screening approach & Target protein & Year & Ref. \\
\hline \multicolumn{5}{|l|}{ Pure-protein screen } \\
\hline IIA4B20 & Binding assay & Myc-Max dimer & 2002 & [53] \\
\hline $\mathrm{BH} 3 \mathrm{l}-1$ & Binding assay & $\mathrm{Bcl}-\mathrm{x}_{\mathrm{L}}$ & 2001 & [54] \\
\hline BTS & Enzymatic assay & Skeletal muscle myosin II & 2002 & [55] \\
\hline PD 098059 & Enzymatic assay & MAP kinase kinase & 1995 & [56] \\
\hline \multicolumn{5}{|l|}{ Cell-based screen } \\
\hline Pifithrin- $\alpha$ & Reporter gene assay & p53 & 1999 & [57] \\
\hline SB 247464 & Reporter gene assay & Granulocyte-colony stimulating factor receptor & 1998 & {$[58]$} \\
\hline Synstab A & Whole-cell immunoassay & Tubulin & 2000 & [59] \\
\hline Myoseverin & Microscope based & Tubulin & 2000 & [22] \\
\hline Splitomicin & Functional assay & Sir2 & 2002 & [40] \\
\hline Monastrol & Whole-cell immunoassay & Mitotic kinesin Eg5 & 1999 & [41] \\
\hline
\end{tabular}




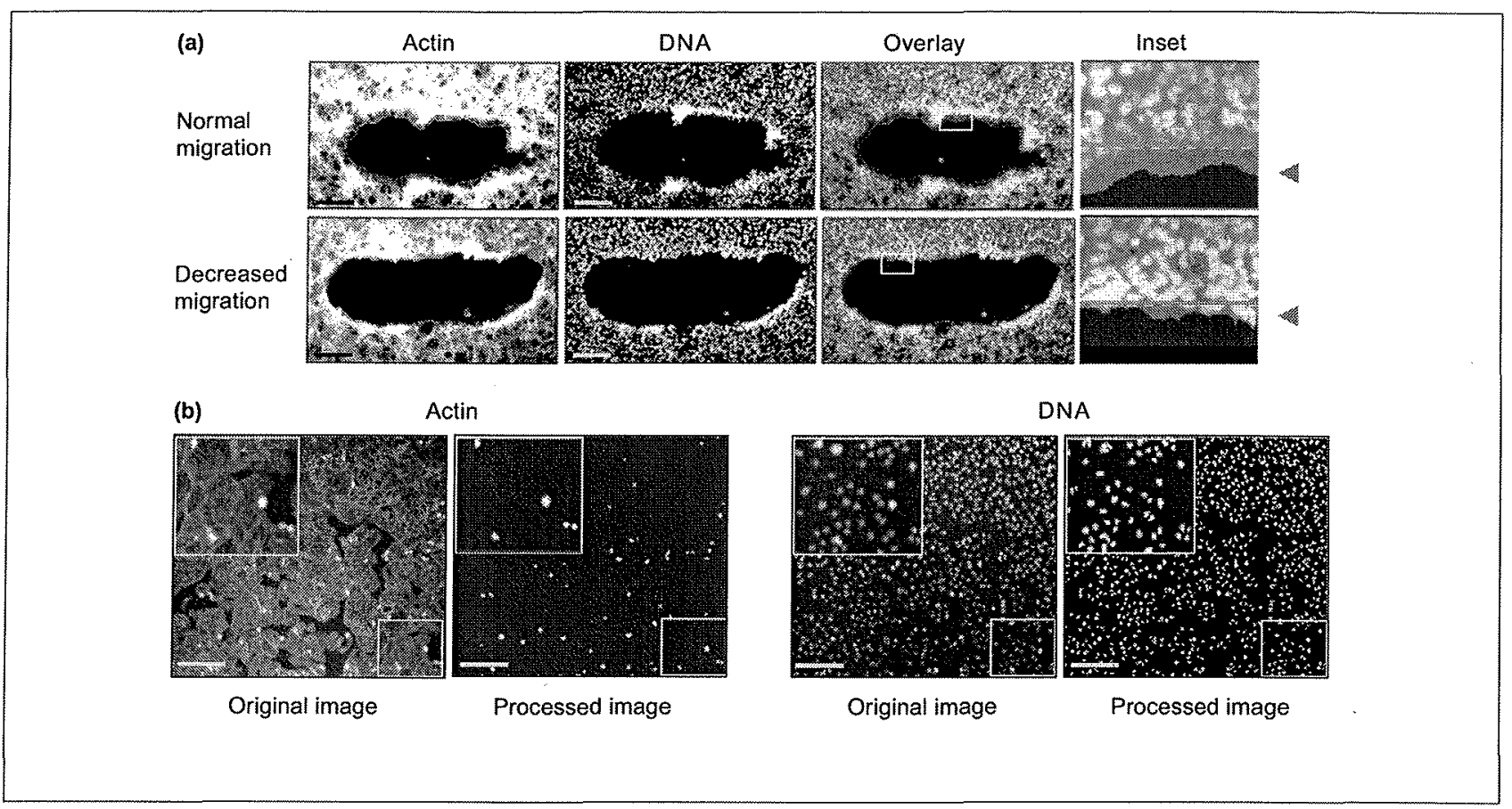

Fig. 3. Microscope-based approaches are capable of detecting subtle changes in cell morphology. \{a\} Immunofluorescence images of wounded cell monolayers. A con. fluent layer of cells in a microtitre plate was mechanically wounded, incubated with compounds and then fixed and stained for the actin cytoskeleton and DNA. The black area represents the induced wound. In the overlay panel, DNA is shown in green, actin in red and colocalization of DNA and actin in yellow. Most right panels show a higher magnification of the inset depicted in the overlay panels. Untreated cells (upper panel) should respond to the wound application by polarized growth, and thereby the distance between the cell periphery (red arrowhead) and the nuclei (blue line) should increase. If compound treatment (lower panel) inhibits polarized growth, this distance should remain constant. Image-analysis algorithms are available to calculate the surface area, which stains for actin but not for DNA (area of polarized growth), thereby providing a quantitative readout for cell migration. (b) Immunofluorescence images of an asynchronous cell population fixed and stained for the actin cytoskeleton and DNA. Computational analyses of the actin image allow the identification and quantification of mitotic cells, based on their round cell morphology. Additionally, the processed DNA image can be used to determine the total cell count per well, supplying information about cell loss owing to manipulation artifacts or putative cytotoxic compounds. Bar, $250 \mu \mathrm{m}$.

when the small molecule lacks a sufficiently high binding affinity for the isolation of target proteins that are only present in low abundance.

By applying cDNA expression-based methods such as phage display [25], protein microarrays [26] or yeast 'three-hybrid systems' [27], the abundance of the target protein can be artificially increased. Phage display methods and protein microarrays follow the same basic concept (see Box 1): a tagged compound (see above) is used as a probe to identify its binding partner within a collection of spatially separated proteins. The basis of the yeast three-hybrid system is a synthetic hybrid molecule composed of a compound with a known binding partner (e.g. dexamethasone, which binds to the hormone-binding domain of the glucocorticoid receptor) and the small molecule of interest (see Fig. I in Box 1). Under conditions where one part of

Table 2. Examples of cellular targets of bioactive small molecules that have been identified by classical biochemical approaches

\begin{tabular}{|lll|}
\hline Compound name & Target protein & Ref. \\
\hline Aminopurvalanol & Cyclin-dependent kinase & {$[32]$} \\
Dihydroeponemycin & 20S proteasome & {$[60]$} \\
Fumagillin & Methionine aminopeptidase & {$[61]$} \\
Ilimaquinone & S-adenosylhomocysteinase & {$[62]$} \\
Trapoxin & Histone deacetylase & {$[63]$} \\
Lactacystin & 20S proteasome & {$[64]$} \\
Rapamycin & FK506 binding protein 12 (FKBP12) & {$[65]$} \\
\hline
\end{tabular}

the hybrid molecule (dexamethasone) binds to its known ligand fused to the DNA-binding domain and the other part (small molecule of interest) binds at the same time to its target fused to the activation domain, transcription will be activated, enabling the expression of a reporter gene (e.g. an essential metabolic enzyme).

New genetic-based approaches for target identification became applicable by the advent of whole-genome sequence information. One possible approach, termed the haploinsufficiency method, follows the logic that lowering the gene dosage from two copies down to one copy sensitizes yeast cells to the drug that acts on the gene product. Recently, this approach was validated on known targets of six small molecules [28]. An alternative approach, which is applicable to yeast as well as to higher eukaryotes, is based on the rationale that increasing the gene dosage of the target gene renders cells more resistant to the compound [29]. A key advantage of these genetic approaches is that they do not require chemical modifications of the selected compound.

At the end of any chemical screen, selected compounds have to pass the acid test of specificity and selectivity. In a first attempt, specificity can be assessed by testing whether the compound modifies the activity of target-related proteins in vitro. But no matter how many proteins are tested in vitro, the risk remains that the assumed target is not the relevant binding partner in vivo. Therefore, additional experiments are required to validate the target. In the case 
of small molecules with an inhibitory mode of action, one possible strategy is to test whether the compound-induced phenotype can be either mimicked by shutting down the activity of the suspected target protein (by biochemical or genetic methods such as immunodepletion, antibody addition or RNA interference [30]) or rescued by artificially increasing the concentration of the suspected target in the assay $[31,32]$.

Recent advances in transcriptional profiling paved the way for compound specificity studies in a global, cellular context [33]. Marton and colleagues applied this approach to show that 3-aminotriazole (3-AT), an inhibitor of His $3 p$ (an enzyme involved in histidine biosynthesis), causes more than 1000 changes in the expression profile of wild type yeast cells. However, almost no changes in transcript level were observable when yeast cells deleted for HIS3 were treated with 3-AT, indicating that 3-aminotriazole is a specific inhibitor of His3p in yeast cells [34].

What have we learned from use of small molecules?

The application of small molecules continues to have an enormous impact on our understanding of cell biology. There is an almost endless list of examples where small molecules, in combination with genetic screens, has facilitated the dissection of complex cellular processes. A famous example is the use of the microtubule poison benomyl to arrest yeast cells at the G2-M transition in a forward-genetic screen designed to identify genes involved in cell-cycle control $[35,36]$. However, the value of small molecules in answering cell-biological questions is clearly not limited to their ability to synchronize cultured cells [37]. Even in Saccharomyces cerevisiae, one of the most widely used model organisms for genetic manipulations, small molecules have provided insights into fundamental biological processes that have not been accessible by classical genetic approaches. For example, the actindepolymerizing activity of latrunculin A, a natural product isolated from the sponge Latrunculia magnifica, has been used to unravel the role of the actin cytoskeleton in cell polarity and mitotic spindle orientation in budding yeast [38] (for review, see [39]). Similarly, the use of splitomicin demonstrated for the first time that continuous histone deacetylase activity of Sir $2 p$ is required for maintaining a transcriptionally silenced state in nondividing yeast cells [40]. In these two studies, small molecules were more successful than genetic approaches because the available conditional mutants did not possess the required temporal resolution (in the former case) or because conditional mutants were not available (in the latter case).

New and exciting insights into mitosis and its regulation in mammalian cells were facilitated by the discovery of the small molecules monastrol [41] and blebbistatin [42]. Monastrol, initially identified in a whole-cell immunoassay designed to identify compounds that arrest tissue-culture cells in mitosis (see Table 1), causes a remarkable phenotype in that the normal bipolar spindle is replaced by an array of microtubules surrounded by a ring of chromosomes (see Fig. 4). A strikingly similar phenotype had been previously observed when antibodies directed against the mitotic kinesin Eg5 were injected into tissue-culture cells [43]. This suggested that Eg5 could be a potential target of
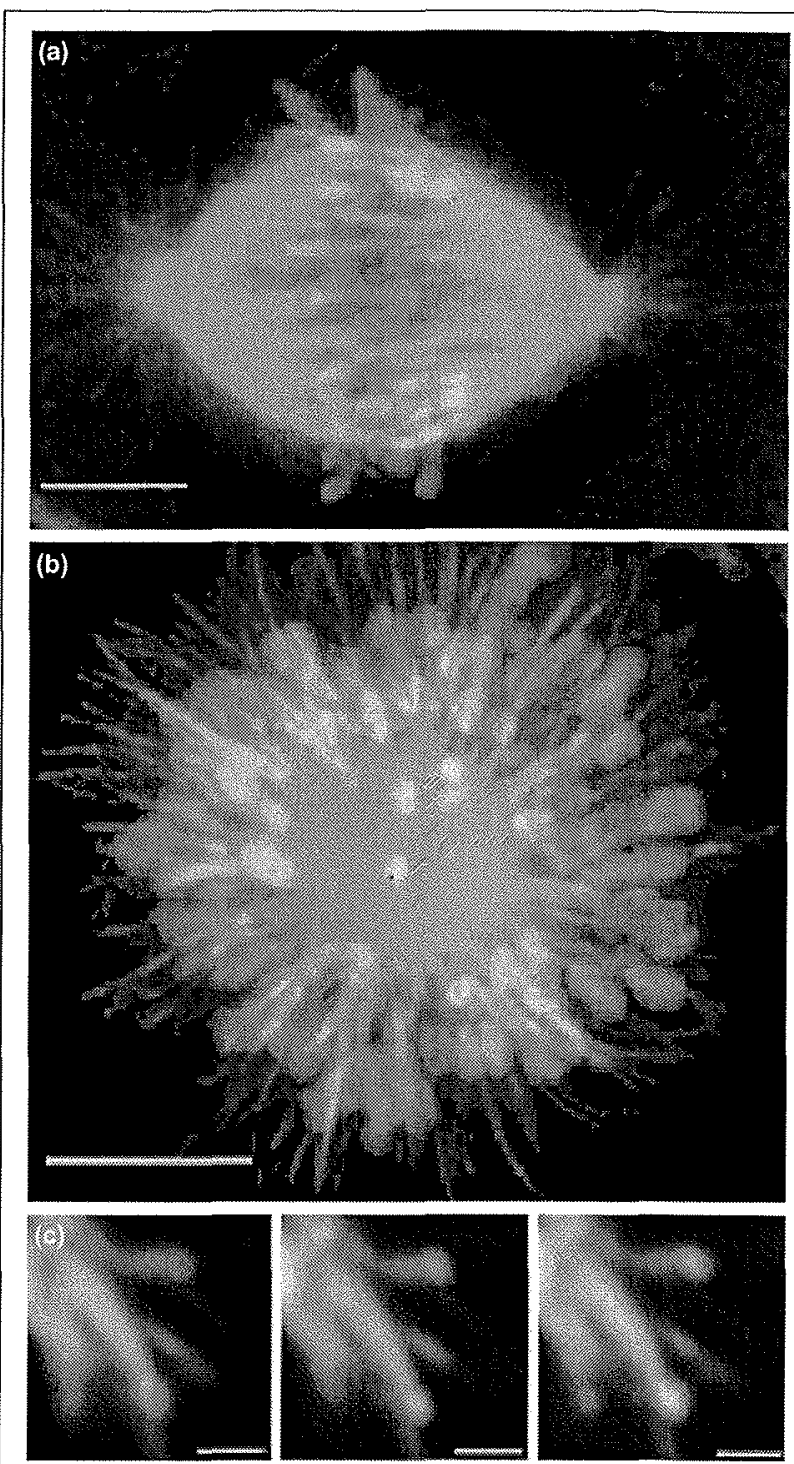

Fig. 4. The small molecule monastrol reveals new insights in the activation of the spindle-assembly checkpoint in tissue-culture cefls. Immunofluorescence images: control-treated (a) and $68 \mu \mathrm{M}$ monastrol-treated (b) African green monkey (BS-C-1) cells stained for DNA (blue) and tubulin (green). in monastrol-treated cells, bipolar spindles do not form but are replaced by monoastral spindles surrounded by a ring of chromosomes. Bar, $5 \mu \mathrm{m}$. (c) Monastrol-treated $(50 \mu \mathrm{m}$ ) rat kangaroo (Ptk-2) cells permeabilized in the presence of $0.1 \mathrm{mM} \mathrm{Ca}^{2+}$ (to clear off all non-kinetochore microtubules) before fixation were stained for tubulin (green), kinetochores (red) and MAD2 (blue). Bar, $1 \mu \mathrm{m}$. Note that the checkpoint protein MAD2 localizes to kinetochores that are attached to microtubules in monoastral spindles. Images adapted, with permission, from Ref. [41] $(a, b)$ and Ref. [44] (c)

monastrol, and indeed motility assays demonstrated that monastrol inhibits Eg5-dependent movement of microtubules in vitro. Owing to its fast mode of action, specificity and reversibility $[41,44]$, monastrol opened up new opportunities to elucidate the function of Eg5 in mitosis. Particular, the provocative idea of the existence of a static spindle matrix re-emerged with the observation that, in Xenopus extracts, Eg5 remains static within the spindle when its motor activity is inhibited by monastrol, whereas tubulin, building up the mitotic spindle, constantly moves 
polewards [45]. Experiments addressing the question of why monastrol-treated cells arrest in mitosis showed that the checkpoint protein MAD2 localizes to a subset of kinetochores in monastrol-treated cells, suggesting the spindle assembly checkpoint is activated in these cells [44]. Moreover, closer examination of monastrol-treated cells revealed MAD2 staining of kinetochores that are clearly attached to microtubules (see Fig. 4). This observation challenges the proposal that microtubule attachment rather than tension regulates association of MAD2 with mammalian kinetochores [46].

Blebbistatin was identified in a pure-protein screen for inhibitors of non-muscle myosin II, which provides the force for the contraction of the cleavage furrow (a ring composed of actin and actin-binding proteins such as myosin II and anillin) during cytokinesis. In vivo, blebbistatin rapidly inhibits the ingression of the cleavage furrow, causing cells to arrest at the end of mitosis with unseparated daughter cells. By adding nocodazole to blebbistatin-treated cells, Straight et al. could demonstrate that microtubule-to-cortex communication is continuously required to maintain the localization of myosin II and anillin at the cleavage furrow. Further experiments using blebbistatin in combination with proteasome inhibitors revealed that exit from cytokinesis in mammalian cells depends on ubiquitin-dependent proteolysis.

Novel insights into the regulation of programmed cell death (apoptosis) were facilitated by the recent discovery of the small molecule PETCM [ $\alpha$-(trichloromethyl)-4pyridine-ethanol] [47]. Wang and coworkers identified PETCM in a high-throughput screen for activators of caspase-3 in an in vitro cell death assay. Searching for the proteins that mediate the PETCM pro-apoptotic effect, Wang and colleagues identified the oncoprotein prothymosin- $\alpha$ (ProT) and the tumor-suppressor putative HLA-DR-associated proteins (PHAPs) as important regulators of caspase-3 activation, albeit with opposing effects. PHAP proteins appear to be positive regulators of apoptosis, whereas Pro $T$ has an anti-apoptotic function by inhibiting the formation of the apoptosome (a macromolecular complex required for the activation of caspase-9, which in turn activates caspase-3). Further experiments demonstrated that PETCM inhibits the anti-apoptotic activity of ProT and thereby promotes the formation of active caspase- 3 in the in vitro cell-death assay. The antiapoptotic activity of ProTwas confirmed by the observation that depletion of ProT by RNA interference sensitizes cultured cells to apoptosis.

A whole collection of small molecules, including brefeldin A, nocodazole, ilimaquinone and okadaic acid, has facilitated important insights into the biology of the Golgi apparatus (reviewed in [48]). These compounds were instrumental, for example, in establishing the cytoskeletal anchoring of the Golgi apparatus close to the MTOC (nocodazole) and in unraveling retrograde flow mechanisms in the secretory pathways (brefeldin A). Similarly, tunicamycin and related inhibitors facilitated the dissection of glycoprotein biosynthesis in a wide range of experimental systems (reviewed in [49]). In these and many other fields of cell biology, small molecules constitute well-established experimental tools to address important problems that are often difficult to approach by other means. In other fields, however, such as neurobiology, developmental biology or cellular microbiology, the use of small molecules has only just begun [50,51]. One example showing the value of chemical genetics in neurobiology is provided by the recent study on the function of myosin-1c in adaptation in hair cells (the process by which the ear's sensitivity to sound is changed by the presence of a constant sound) [52]. By expressing a mutant form of myosin-1c susceptible to inhibition by N6-modified ADP analogs in utricular hair of transgenic mice, Holt et al. were able to show that the ADP analog largely blocks the ability of these cells to adapt to mechanical stimuli, suggesting that myosin-1c participates in adaptation in hair cells. This inhibition was specific for the engineered mysoin-1c because adaptation in transgenic mice expressing wild-type Myo-1c was entirely normal, even in the presence of the ADP analog.

\section{Concluding remarks}

There is no doubt that small molecules have proven themselves as invaluable tools for cell biology. However, it is important to bear in mind that the value of chemical genetics is inseparably tied to the quality of the applied molecules, with compound specificity representing the most crucial issue. In the light of this challenge, a combined effort on the part of chemists and biologists is required to generate novel, highly diverse libraries of small molecules, to design suitable screening approaches and finally to validate the binding partners of active compounds as the relevant targets in vivo. Given the enormous progress that has been made in each of these fields, chemical genetics will have a rapidly growing impact on modern biology.

\section{Acknowledgements}

I thank Erich Nigg, Olaf Stemmann, Alison Dalvofo, and members of my lab for critical comments on the manuscript and Justin Yarrow for Fig. 3a. T.U.M. is funded by the Emmy Noether Program of the Deutschen Forschungsgemeinschaft (DFG).

\section{References}

1 Hartwell, L.H. (1991) Twenty-five years of cell cycle genetics, Genetics 129, 975-980

2 Brenner, S. (1974) The genetics of Caenorhabditis elegans. Genetics 77, $71-94$

3 Nusslein-Volhard, C. and Wieschaus, E. (1980) Mutations affecting segment number and polarity in Drosophila. Nature 287, 795-801

4 Bishop, A. et al. (2000) Unnatural ligands for engineered proteins: new tools for chemical geneties. Annu. Rev. Biophys. Biomol. Struct. 29, $577-606$

5 Klausner, R.D. et al. (1992) Brefeldin A: insights into the control of membrane traffic and organelle structure. J. Cell Biol. 116, 1071-1080

6 Brinkley, B.R. et al. (1967) The effects of colcemid inhibition and reversal on the fine structure of the mitotic apparatus of Chinese hamster cells in vitro. J. Ultrastruct. Res. 19, 1-18

7 Borisy, G.G. and Taylor, E.W. (1967) The mechanism of action of colchicine. Binding of colchincine- $3 \mathrm{H}$ to cellular protein. J. Cell Biol. $34,525-533$

8 Avila, J. (1992) Microtubule functions. Life Sci. 50, 327-334

9 Khayat, D. et al. (2000) Taxol in the management of cancers of the breast and the ovary. Cancer Invest. 18, 242-260

10 Schiff, P.B. et al. (1979) Promotion of microtubule assembly in vitro by taxol. Nature 277,665-667 
11 Schiff, P.B and Horwitz, S.B. (1980) Taxol stabilizes microtubules in mouse fibroblast cells. Proc. Natl. Acad. Sci. U. S. A. 77, 1561-1565

12 Vallee, R.B. (1982) A taxol-dependent procedure for the isolation of microtubules and microtubule-associated proteins (MAPs). J. Cell Biol. 92, 435-442

13 Cohn, S.A. et al. (1993) Analyzing microtubule motors in real time. Methods Cell Biol. 39, 75-88

14 Dolle, R.E. (2002) Comprehensive survey of combinatorial library synthesis: 2001. J. Comb. Chem. 4, 369-418

15 On the World Wide Web URL: http://www.combichem.net

16 Muegge, I. (2002) Pharmacophore features of potential drugs Chemistry 8, 1976-1981

17 On the World Wide Web URL: http://tp.nci.nih.gov/webdata.html http://www.ninds.nih.gov/funding/neurodegeneration/NINDS__Drug_ Screening.htm, http://www.hki-jena.de/hki hp.htm

18 Hertzberg, R.P. and Pope, A.J. (2000) High-throughput screening: new technology for the 21st century. Curr. Opin. Chem. Biol. 4, 445-451

19 Stockwell, B.R. et al. (1999) High-throughput screening of smal molecules in miniaturized mammalian cell-based assays involving post-translational modifications. Chem. Biol. 6, 71-83

20 Boland, M.V. et al. (1998) Automated recognition of patterns characteristic of subcellular structures in fluorescence microscopy images Cytometry 33, 366-375

21 Peterson, R.T. et al. (2000) Small molecule developmental screen reveal the logic and timing of vertebrate development. Proc. Natl. Acad. Sci. U. S. A. 97, 12965-12969

22 Rosania, G.R. et al. (2000) Myoseverin, a microtubule-binding molecule with novel cellular effects. Nat. Biotechnol. 18, 304-308

23 Pelish, H.E. et al. (2001) Use of biomimetic diversity-oriented synthesis to discover galanthamine-like molecules with biological properties beyond those of the natural product. J. Am. Chem. Soc. 123 $6740-6741$

24 Mitchison, T.J. (1994) Towards a pharmacological genetics. Chem. Biol. 1, 3-6

25 Tanaka, A.S. et al. (1999) Functional phage display of leech-derived tryptase inhibitor (LDTI): construction of a library and selection of thrombin inhibitors. FEBS Lett. 458, 11-16

26 MacBeath, G. and Schreiber, S.L. (2000) Printing proteins as microarrays for high-throughput function determination. Science $289,1760-1763$

27 Licitra, E.J. and Liu, J.O. (1996) A three-hybrid system for detecting small ligand-protein receptor interactions. Proc. Natl. Acad. Sci U. S. A. 93, 12817-12821

28 Giaever, G. et al. (1999) Genomic profiling of drug sensitivities via induced haploinsufficiency. Nat. Genet. 21, 278-283

29 Launhardt, H. et al. (1998) Drug-induced phenotypes provide a tool for the functional analysis of yeast genes. Yeast 14, 935-942

30 Wignall, S.M. et al. (2000) A chemistry-based approach to identify regulators of spindle assembly: Characterization of a microtubule destabilizing compound and target identification. Mol. Biol. Cell 11 $361 a$

31 Casaubon, R.L and Snapper, M.L (2001) S-adenosylmethionine reverses ilimaquinone's vesiculation of the Golgi apparatus: a fluorescence study on the cellular interactions of ilimaquinone. Bioorg. Med. Chem. Lett. 11, 133-136

32 Rosania, G.R. et al. (1999) A cyclin-dependent kinase inhibitor inducing cancer cell differentiation: biochemical identification using Xenopus egg extracts. Proc. Natl. Acad. Sci. U. S. A. 96, 4797-4802

33 Butte, A. (2002) The use and analysis of microarray data. Nat. Rev. Drug Discov. 1, 951-960

34 Marton, M.J. et al. (1998) Drug target validation and identification of secondary drug target effects using DNA microarrays. Nat. Med. 4 $1293-1301$

35 Li, R. and Murray, A.W. (1991) Feedback control of mitosis in budding yeast. Cell $66,519-531$

36 Hoyt, M.A. et al. (1991) S. cerevisiae genes required for cell cycle arrest in response to loss of microtubule function. Cell $66,507-517$

37 Crews, C.M. and Mohan, R. (2000) Small-molecule inhibitors of the cell cycle. Curr. Opin. Chem. Biol. 4, 47-53
38 Theesfeld, C.L et al. (1999) The role of actin in spindle orientation changes during the Saccharomyces cerevisiae cell cycle. J. Cell Biol. 146, 1019-1032

39 Peterson, J.R. and Mitchison, T.J. (2002) Small molecules, big impact. A history of chemical inhibitors and the cytoskeleton. Chem. Biol. 9 $1275-1285$

40 Bedalov, A. et al. (2001) Identification of a small molecule inhibitor of Sir2p. Proc. Natl. Acad. Sci. U. S. A. 98, 15113-15118

41 Mayer, T.U. et al. (1999) Small molecule inhibitor of mitotic spindle bipolarity identified in a phenotype-based screen. Science 286 971-974

42 Straight, A.F. et al. (2003) Dissecting temporal and spatial control of cytokinesis with a myosin II inhibitor. Science 299, 1743-1747

43 Blangy, A. et al. (1995) Phosphorylation by $\mathrm{p} 34 \mathrm{cdc} 2$ regulates spindle association of human $\mathrm{Eg} 5$, a kinesin-related motor essential for bipolar spindle formation in vivo. Cell 83, 1159-1169

44 Kapoor, T.M. et al. (2000) Probing spindle assembly mechanisms with monastrol, a small molecule inhibitor of the mitotic kinesin, Eg5. J. Cell Biol. 150, 975-988

45 Kapoor, T.M. and Mitchison, T.J. (2001) Eg5 is static in bipolar spindles relative to tubulin: evidence for a static spindle matrix. J. Cell Biol $154,1125-1133$

46 Waters, J.C. et al. (1998) Localization of Mad2 to kinetochores depends on microtubule attachment, not tension. $J$. Cell Biol. 141, 1181-1191

47 Jiang, X. et al. (2003) Distinctive roles of PHAP proteins and prothymosin-alpha in a death regulatory pathway. Science 299 , 223-226

48 Dinter, A. and Berger, E.G. (1998) Golgi-disturbing agents. Histochem. Cell Biol. 109, 571-590

49 McDowell, W. and Schwarz, R.T. (1988) Dissecting glycoprotein biosynthesis by the use of specific inhibitors. Biochimie 70, 1535-1549

50 Stockwell, B.R. (2002) Chemical genetic screening approaches to neurobiology. Neuron $36,559-562$

51 Ward, G.E. et al. (2002) Using small molecules to study big questions in cellular microbiology. Cell Microbiol. 4, 471-482

52 Holt, J.R. et al. (2002) A chemical-genetic strategy implicates myosin-1c in adaptation by hair cells. Cell 108, 371-381

53 Berg, T. et al. (2002) Small-molecule antagonists of Myc/Max dimerization inhibit Myc-induced transformation of chicken embryo fibroblasts. Proc. Natl. Acad. Sci. U. S. A. 99, 3830-3835

54 Degterev, A. et al. (2001) Identification of small-molecule inhibitors of interaction between the BH3 domain and Bcl-xL. Nat. Cell Biol. 3 $173-182$

55 Cheung, A. et al. (2002) A small-molecule inhibitor of skeletal muscle myosin II. Nat. Cell Biol. 4, 83-88

56 Dudley, D.T et al. (1995) A synthetic inhibitor of the mitogen-activated protein kinase cascade. Proc. Natl. Acad. Sci. U. S. A. 92, 7686-7689

57 Komarov, P.G. et al. (1999) A chemical inhibitor of p53 that protects mice from the side effects of cancer therapy. Science 285, 1733-1737

58 Tian, S.S. et al. (1998) A small, nonpeptidyl mimic of granulocytecolony-stimulating factor. Science 281, 257-259

59 Haggarty, S.J. et $\alpha$ l. (2000) Dissecting cellular processes using smal molecules: identification of colchicine-like, taxol-like and other small molecules that perturb mitosis. Chem. Biol. 7, 275-286

60 Meng, L. et al. (1999) Eponemycin exerts its antitumor effect through the inhibition of proteasome function. Cancer Res. 59, 2798-2801

$61 \mathrm{Sin}, \mathrm{N}$. et al. (1997) The anti-angiogenic agent fumagillin covalently binds and inhibits the methionine aminopeptidase, MetAP-2. Proc. Natl. Acad. Sci. U. S. A. 94, 6099-6103

62 Radeke, H.S. and Snapper, M.L. (1998) Photoaffinity study of the cellular interactions of ilimaquinone. Bioorg. Med. Chem. 6, 1227-1232

63 Taunton, J. et al. (1996) A mammalian histone deacetylase related to the yeast transcriptional regulator Rpd3p. Science 272, 408-411

64 Fenteany, G. et al. (1995) Inhibition of proteasome activities and subunit-specific amino-terminal threonine modification by lactacystin. Science 268, 726-731

65 Harding, MW et al (1989) A receptor for the immunosuppressan FK506 is a cis-trans peptidyl-prolyl isomerase. Nature $341,758-760$

66 Ab-Obeidi, F. et al. (1998) Peptide and peptidomimetic libraries. Molecular diversity and drug design. Mol. Biotechnol. 9, 205-223 